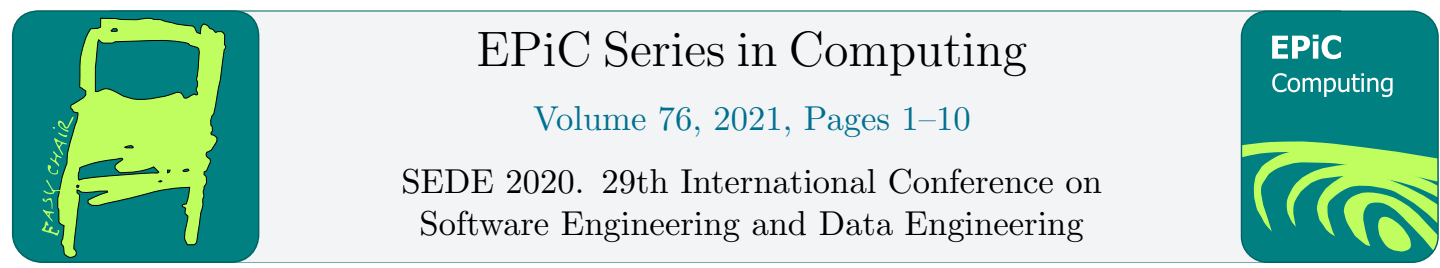

\title{
Cloud Applications Adoption: User Study Survey From Industry and Academia
}

\author{
Jalal H. Kiswani ${ }^{1}$, Sergiu M. Dascalu ${ }^{2}$, and Frederick C Harris, Jr. ${ }^{3}$ \\ 1 Department of Computer Science and Engineering \\ University of Nevada, Reno, \\ Reno, NV, USA \\ jalal@nevada.unr.edu \\ 2 Department of Computer Science and Engineering \\ University of Nevada, Reno, \\ Reno, NV, USA \\ dascalus@cse.unr.edu \\ 3 Department of Computer Science and Engineering \\ University of Nevada, Reno, \\ Reno, NV, USA \\ fred.harris@cse.unr.edu
}

\begin{abstract}
Cloud computing has changed the ways software systems are developed, deployed, and managed. Even though cloud computing could reduce cost, time to market, and increase the scalability of applications and systems, there are some debates about the actual adoption of cloud computing in general and cloud applications in particular by organizations from both, industry and academia, and whether stakeholders of organizations look for them as opportunities or risks. To answer these debates, we conducted a user study that included professionals from industry and academia, with various experience levels and job-roles, to be able to get a common understanding of the real value of cloud computing. In particular, the study included questions about the preferred approach of developing green-field applications and the availability of resources and expertise. In addition, it included questions about the cost of development, maintainability, and operations of cloud applications. The results of the user study are presented and discussed, and we believe that it is promising and could be used as the base of future directions of research and technology innovation.
\end{abstract}

\section{Introduction}

There is no doubt that cloud computing is being utilized heavily around the globe by organizations and entities on all levels. It can be seen everywhere from online shopping websites, such as Amazon and Walmart, to the media industry such as NetFlix and HBO, to the transportation domain such as Uber and Lyft; where most of these services, probably, wouldn't be offered at these scales without cloud computing. Furthermore, cloud computing has enabled 
a lot of startup companies to have their services offered in a very short time compared to the traditional approaches of deploying their systems and implementing external services. In fact, the entrepreneurship ecosystems wouldn't be the same without it.

However, as any new innovation and trend, the maturity of technologies and tools that enables the full potential of cloud computing may require some time [1]. In particular, cloud computing and the various categories of the services offered under its umbrella (i.e. IaaS, PaaS, and SaaS) explained in Section II, are all still evolving and being improved day after day to exploit the full potential and traction.

In fact, and based on the report published by RightScale in 2018, and as shown in Figure 1, resources availability and cost are two of the top barriers of adopting cloud computing in organizations [2]. Other barriers include: security, managing cloud spends, governance and control, compliance, management of multiple clouds, performance, and building a private cloud.

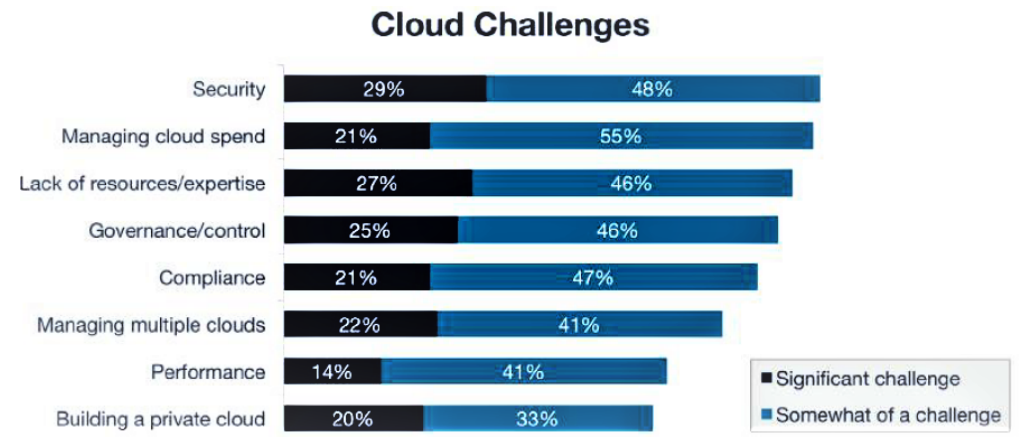

Figure 1: Top cloud barriers [2]

While these barriers are common to all cloud computing services offering, we wanted to understand if organizations and professionals prefer to develop new applications using the cloudbased approach, that utilizes the full benefits of cloud computing, or the traditional on-premise approach. This question applies to both green-field (i.e. new projects with minimum constraints) and brown-field projects (i.e. highly constrained projects with factors such as legacy systems). Moreover, we wanted to analyze their perspective of the availability-of-resources barrier; in addition to the cost of development, operation, and maintenance. Furthermore, we wanted to hear feedback about migrating legacy applications to a new cloud-based approach.

To be able to get answers to all these questions, we conducted a user study that included 36 professionals from industry and academia. In the study, we included participants with years of experience ranging from 3 to 20 years, and with various job roles, such as software developers, system architects, directors, and professors. The main reason behind this diversity was to be able to get an understanding that is representative as much as possible.

The understanding of the behavior and thoughts of organizations and people in regards to cloud computing may help both researchers and service providers identify the areas that could be enhanced to increase the adoption of cloud computing services and cloud-applications approach, which may enable their full potential.

This paper is organized into five sections. This section includes the introduction. Section II includes the background and literature review. Section III includes the user study general information. Section IV includes the results and discussion. Finally, Section V concludes this paper and identifies future directions. 


\section{Background and Literature Review}

Cloud computing is the new approach of offering various computing-related services as a subscription-based pay-as-you-go approach (i.e., renting) instead of owning them (i.e., buying). In a subscription-based approach, customers can get services for a fraction of what they pay for the perpetual buying-approach. On the other hand, service providers could benefit from having a stable revenue streams based on the economies of scale business model; in particular, having small profit margins for a large customers base.

While there are different categorizations of cloud-computing services, the main classification is Infrastructure as a Service (IaaS), Platform as a Service (PaaS), and Software as a Service (SaaS) or cloud applications [3].

The infrastructure service offering is based on groups of data centers offered by cloud providers to serve cloud users. At the top of these data centers there is virtualization platforms that enables them to be shown as a pool of resources that could be accessed transparently without the need of knowing or managing the underlying physical infrastructure. These resources could be accessed publically, by a specific group of entities, or a private entity, in what so-called public, hybrid, and private cloud respectively [4].

On the other hand, the platform service offerings provide the required infrastructure and tools for cloud-users to enable them to build and develop software systems over these services. For example, for a parallel programming task based on MPI C-language library, a platform could provide the nodes that include the master and workers, the network between them, and the programming language compiler, tools, and MPI library to achieve the task. In this case, the cloud customers focus only on the programming part, and will have all the infrastructure ready for them out of the box.

The software services of cloud computing are based on having a software system deployed and ready to be used by service users [5]. For example, in an Enterprise Resource Planning System (ERP), normally, infrastructure (e.g., multiple servers and network), platforms (e.g., database servers and application servers), and implementation are required to have the system up and ready to be used by the end-users, which normally takes a long time, maybe months, while it could be done in hours, or sometimes minutes, using the SaaS approach.

Software applications in general, and cloud applications in particular, could be developed in different architectural styles, the monolithic or the microservices-based [6].

In the monolithic approach, the software is built as a big chunk of software that could only be deployed as a single unit [7]. This approach has been used for decades now, since it is convenient and easy to develop, debug, and deploy. However, with the increasing size of software systems, the rise of internet businesses, and the need for faster time to market, the monolithic approach is not sufficient to face the new challenges, where maintainability, scalability, and availability quality-attributes have been true challenges. Furthermore, new trends such as global software engineering, Continuous Integration (CI), Continuous Delivery (CD), agility, and the digital transformation wave in organizations require a new approach of building software systems, the microservices approach.

In the microservices approach, software systems are decomposed into smaller components (i.e., microservices) that are independently deployable, built-around business capabilities, and could be developed in any technology [8]. In addition, systems could be developed incrementally, where components could be deployed as they are built, without any technology, and from any location. Moreover, every microservice shall manage its own data, where the technical coupling between the services is the minimum, and any required data is exposed through the service itself, not the database. 
Even though the microservices approach has been widely adopted over the last decade, it has introduced a level of architectural and operational complexity that put a lot of projects under the risk of failure. In particular, the design, orchestration, and scalability of microservices required new techniques and tools; in fact, based on Martin's Fowler recommendation, it is safer to start by following the monolithic approach [9].

However, not all projects have the same characteristics and constraints, some could start just from the beginning (i.e. green-field), or could be a continuity of enhancement, or may be replacement of previous, and probably legacy system (i.e. brown-field). While the green-field case is the preferred one for technical people and management, since it has lower constraints; life is not always green, and neither are projects in most of the organizations. In most organizations, and mainly medium to large size organizations, there could be a lot of constraints related to other systems and regulations that may increase the complexity and reduce the flexibility of the decisions that could be made to build high-quality systems [10].

While there are some researches that study the adoption of cloud computing in organizations such as RightScale [2], and to the best of our knowledge, we did not find a user study that covers the cloud-application based approach adoption by individual practitioners and researchers from both, the academia and the industry perspectives.

\section{User Study}

This section includes the details of the user study conducted to analyze the adoption of cloud computing and cloud applications in organizations from different levels.

\subsection{Methodology}

The user study is based on a survey conducted between the period of 24 to 31 December, 2018 . It included 36 software technology practitioners and academics with various seniority levels, positions, and education. In addition, the participants come from different regions all over the world.

The user study survey was organized into three sections: Introductory Information, Participant Information, and Cloud Applications.

- Introductory Information: In the survey, this section included an introduction about the user study, so that participants could understand the goals. Then, it followed by a consent required by the Institutional Review Boards (IRB) process of university of Nevada, Reno (UNR). After that, it included the email address of the participant, then (optionally) his/her name. Finally, we asked them if we could contact them for future feedback or questions if needed.

- Participant Information: The second section, aimed to get general information about the participants themselves to ensure that we have a representative group. The information questions included the participant's overall experience in the software development field, highest academic degree, current job title, years of experience, and the day-to-day activities working on software development projects.

- Cloud Applications: This section included questions about cloud applications significance, cost, and limitations. In particular, the first question was whether choosing cloud applications is more beneficial than the traditional approaches for green-field projects. The second question is to verify if there is a shortage in experienced software engineers who 
can develop high-quality cloud-based applications. The third, fourth, and fifth questions were about the cost of software development, maintenance, and operation of cloud versus traditional applications respectively. The last question in this section was about the risk of uplifting traditional applications (i.e., migrating applications to the cloud).

\subsection{Participants}

As shown in Figure 2, the survey included a wide range of participants with different professional levels in cloud computing. In particular, around $33 \%$ are professionals, $36 \%$ are intermediate, and $27 \%$ are experts in the field of study.

\section{What is your experience level in the software development field?}

\section{6 responses}

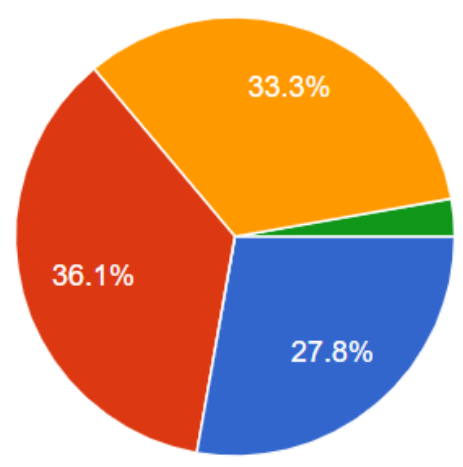

Expert level

Professional level

Intermediate level

Entry level

Figure 2: Experience levels of the user study participants

In addition, the participants came from different academic backgrounds, as shown in Figure 3. Where around $55 \%$ have a bachelor degree, $30 \%$ with Masters degree, and around $14 \%$ with a $\mathrm{PhD}$ degree.

Moreover, Figure 4 shows the participants day-to-day involvement in software development projects activities. So that we can get a clear understanding of the different point of views. The day involvement categories included: research, project management, business analysis, software architecture, software development, software implementations, testing, teams management, technical support, and teaching or training. As shown in the figure, the categories included participants with $72 \%$ of software development, $44 \%$ teams management and software architecture, and $50 \%$ software implementation.

Also, we included a question about the job titles of the participants. The participants titles include: Solution Managers, System architects, Associate and Assistant Professors, IT Directors, System Analysts, Ph.D. Students, Implementation Managers, Services Manager, Integration Specialist, Technical Team Leader, Head of QA, Software Engineers, Senior Software Engineers, and Software Development Consultants.

Finally, we asked them about the total years of experience their jobs in the IT field. and the total years of experience of them range from 3-20 years of experience. 


\section{What is your highest academic degree?}

36 responses

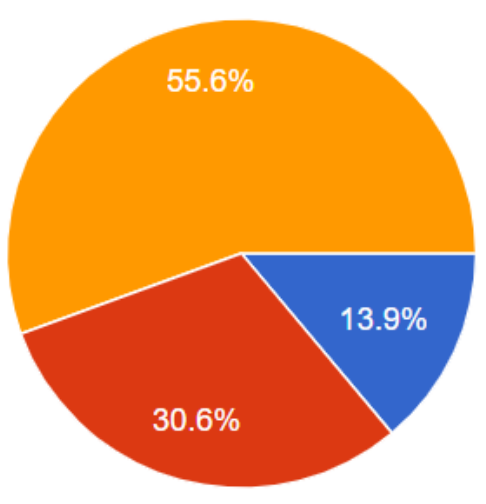

Doctoral

Masters

Bachelor

Figure 3: Academic levels of the participants in the user study

\section{What is your current day to day work in software development projects? (please check all that apply)}

36 responses

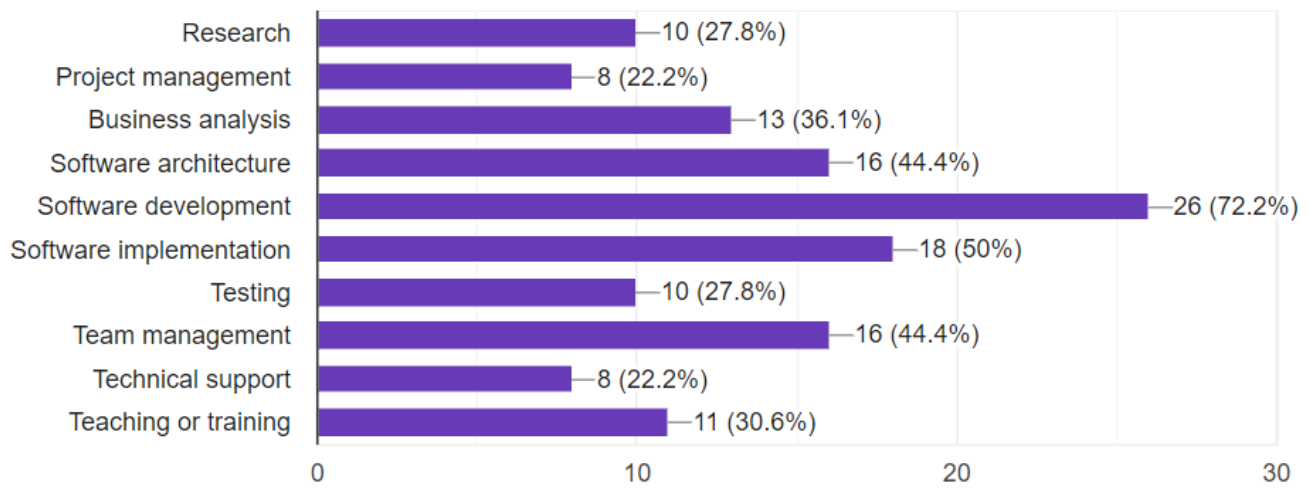

Figure 4: Involvement of the participants in software development activities

\section{Results and Discussion}

This section includes the results of the conducted user study in addition to the results discussion.

The results of the user study are shown in Table 1, which includes the questions and their aggregate responses.

Nowadays, a cloud-based approach of software application development is one of the impor- 
Table 1: The results of the user study

\begin{tabular}{|c|c|c|c|c|c|c|}
\hline $\begin{array}{l}\text { Question } \\
\text { Ranking Value }\end{array}$ & $\begin{array}{l}\text { Strongly } \\
\text { Disagree } \\
\quad(1)\end{array}$ & $\begin{array}{l}\text { Disagree } \\
\text { (2) }\end{array}$ & $\begin{array}{l}\text { Neutral } \\
\text { (3) }\end{array}$ & $\begin{array}{l}\text { Agree } \\
(4)\end{array}$ & $\begin{array}{c}\text { Strongly } \\
\text { Agree } \\
(5)\end{array}$ & Average \\
\hline $\begin{array}{l}\text { For new software applications, choos- } \\
\text { ing a cloud-based approach can be } \\
\text { more beneficial than choosing tradi- } \\
\text { tional approaches }\end{array}$ & - & $1(2.8 \%)$ & $3(8.3 \%)$ & $19(52.8 \%)$ & $11(30.6 \%)$ & 4.18 \\
\hline $\begin{array}{l}\text { There is a shortage of experienced } \\
\text { software engineers who can develop } \\
\text { high-quality cloud applications }\end{array}$ & - & - & $10(27.8 \%)$ & $16(44.4 \%)$ & $10(27.8 \%)$ & 4 \\
\hline $\begin{array}{l}\text { The cost of software development of } \\
\text { cloud based applications is higher } \\
\text { than cost of developing traditional } \\
\text { software }\end{array}$ & $1(2.8 \%)$ & $18(50 \%)$ & $6(16.7 \%)$ & $10(27.8 \%)$ & - & 2.71 \\
\hline $\begin{array}{l}\text { The maintainability cost of cloud } \\
\text { based applications is higher than that } \\
\text { of traditional software applications }\end{array}$ & $4(11.1 \%)$ & $19(52.8 \%)$ & $4(11.1 \%)$ & $6(16.7 \%)$ & $2(5.6 \%)$ & 2.72 \\
\hline $\begin{array}{l}\text { The operational cost of cloud based } \\
\text { applications is higher than that of tra- } \\
\text { ditional software applications }\end{array}$ & $3(8.3 \%)$ & $15(41.7 \%)$ & $5(13.9 \%)$ & $10(27.8 \%)$ & $2(5.6 \%)$ & 2.80 \\
\hline $\begin{array}{l}\text { Migrating applications developed us- } \\
\text { ing traditional approaches to be cloud- } \\
\text { based is an expensive and risky pro- } \\
\text { cess }\end{array}$ & $1(2.8 \%)$ & $5(13.9 \%)$ & $8(22.2 \%)$ & $17(47.2 \%)$ & $2(5.6 \%)$ & 3.42 \\
\hline
\end{tabular}

tant current trends in the software engineering industry. However, we believe that there are many challenges surrounding the adoption of this approach in many organizations.

Before trying to identify these problems, we wanted to be sure that building applications following the cloud approach is the preferred way especially in green-field projects over the traditional approach, in organizations in different domains. So based on the results of the user study, as shown in Figure 5 more than $82 \%$ of the participants answered the question "For new software applications, choosing a cloud-based approach can be more beneficial than choosing a traditional approach" that choosing a cloud application can be more beneficial than the traditional approach in new software projects, with an average of $4.18 / 5$, where 5 is Strongly Agree and 1 is Strongly Disagree.

In addition, and as shown in Table 1 , around $50 \%$ of the participants think that the development, maintainability, and operational cost of cloud applications is lower than traditional approaches. As shown in the table, the average of the responses are 2.71, 2.72, and 2.8 respectively, where lower values means that the cloud is cheaper. The goal behind designing the survey for these questions in an opposite direction is to ensure that the participants are completing the survey with rational data and not rushing the answers.

The above results shows the significance of cloud computing and cloud applications in reducing the cost of development, maintenance, and operations.

However, since averages of development, maintainability, and operational cost are all close to the median, we think that these questions require more investigation.

On the other hand, all the previous questions were related to new projects in a green field situation. So, we also wanted get the particpants opinion about brown-field projects to check if migrating current applications to be cloud-based is considered a risky process for the organizations, and the results show that more than $40 \%$ of the participants think it is risky to uplift on-premises applications (e.g. migrating applications to the cloud), with an average of $3.42 / 5$. However, we also think that this requires more investigation, since based on the feedback of some the participants, privacy, compliance, and security concerns are still dominant.

So the question is, if most of the participants think that cloud applications are more benefi- 


\section{For new software applications, choosing a cloud-based approach can be more beneficial than choosing traditional approaches.}
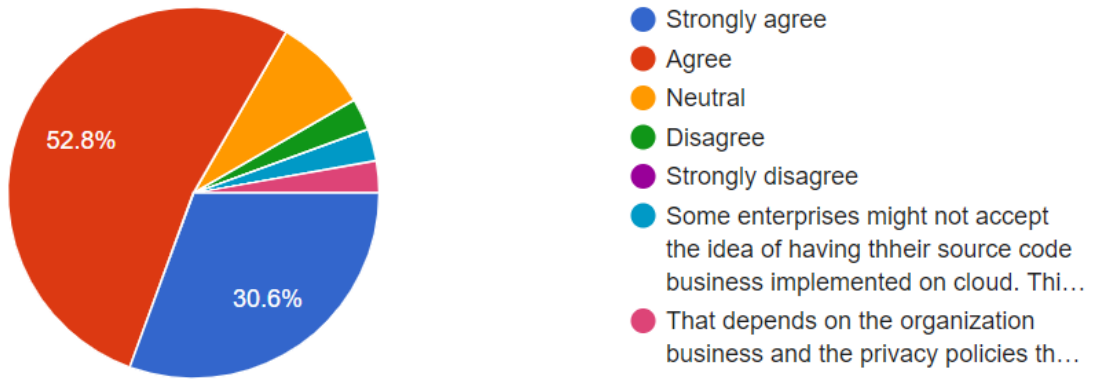

Figure 5: Cloud-based approach versus the traditional approach

cial than the monolithic based, then why there are still many on-premises applications in many organizations? The answer to this question is shown in Figure 6, where more than $71 \%$ of the participants say that there is a shortage in experienced software engineers who can develop high-quality cloud software applications, with an average of $4 / 5$ of the participants think that there is a shortage in this area, which confirms the results of RightScale [2], which discussed in Section I, that is the lack of resources and expertise has become a major barrier for adopting cloud computing. While the results in RightScale report including cloud computing in general, the study shows that it could be also applied in cloud applications in particular.

We believe that the above results, show there is a need for an approach that enables developing cloud-based applications in an efficient and effective way, without requiring particular expertise, is significant and worth attention.

\section{Conclusion and Future Work}

The main goal of this paper was to try to understand the actual adoption of cloud-based approach of developing new software systems in industry and academia and in different domains; in addition, studying the barriers that could affect organization's decision to implement this approach.

The work in this research is based on a user study that was conducted for 36 professionals from academia and the industry from different regions around the globe, expertise, and jobs roles. The user study mainly shows that cloud-applications are the preferred approach for most of the participants and the cost of adopting cloud applications is lower than the traditional one. However, the participants say that the lack of expertise is the main challenge of adopting cloud-based applications.

However, we believe that there is a need to investigate the actual cost of development, maintenance, and operations of cloud-based applications versus the traditional on-premise category, since the results in the user study was close to the median.

In addition, finding an approach that enables effective and efficient development of cloud- 


\section{There is a shortage of experienced software engineers who can develop high-quality cloud applications}

36 responses

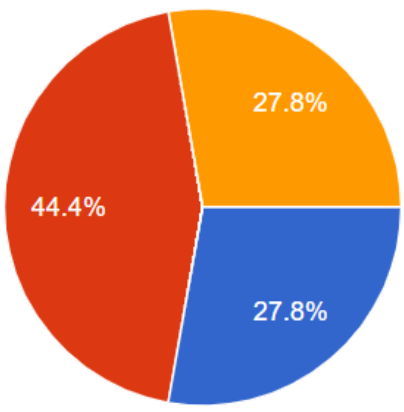

Strongly agree

Agree

Neutral

Disagree

Strongly disagree

Figure 6: Shortage in experienced cloud application's developers

based applications without the need for special expertise will be significant and increase the adoption of cloud computing.

\section{Acknowledgment}

This material is based upon work supported by the National Science Foundation under grant number IIA-1301726. Any opinions, findings, and conclusions or recommendations expressed in this material are those of the authors and do not necessarily reflect the views of the National Science Foundation. The user study was an IRB approved by the University of Nevada, Reno (IRB \#1362116-1).

\section{References}

[1] James Utterback. The dynamics of innovation. Harvard Business School Press, Boston, 1994. https://www . amazon. com/Mastering-Dynamics-Innovation-James-Utterback/dp/0875847404.

[2] RightScale. State of the cloud report, date to navigate your multi cloud strategy. Technical report, RightScale, https://www.rightscale.com/lp/state-of-the-cloud, 2018.

[3] Peter Mell and Tim Grance. The NIST definition of cloud computing, 2011. http://faculty.winthrop.edu/domanm/csci411/Handouts/NIST . pdf.

[4] Michael Armbrust, Armando Fox, Rean Griffith, Anthony D Joseph, Randy H Katz, Andrew Konwinski, Gunho Lee, David A Patterson, Ariel Rabkin, Ion Stoica, and Mate Zaharia. Above the Clouds: A Berkeley View of Cloud Computing. Technical Report UCB/EECS-2009-28, EECS Department, University of California, Berkeley, https://www2. eecs . berkeley . edu/Pubs/TechRpts/2009/EECS-2009-28.pdf, 2009.

[5] Abhijit Dubey and Dilip Wagle. Delivering software as a service. The McKinsey Quarterly, 6(2007):2007, 2007.

[6] Armin Balalaie, Abbas Heydarnoori, and Pooyan Jamshidi. Microservices architecture enables devops: migration to a cloud-native architecture. IEEE Software, 33(3):42-52, 2016. 
[7] Tom Killalea. The hidden dividends of microservices. Communications of the ACM, 59(8):42-45, 2016.

[8] Martin Fowler and James Lewis. Microservices: a definition of this new architectural term, March 2014. (Date last accessed August 15, 2019).

[9] Martin Fowler. Monolithfirst, June 2015. (Date last accessed August 15, 2019).

[10] Carmine Giardino, Nicolo Paternoster, Michael Unterkalmsteiner, Tony Gorschek, and Pekka Abrahamsson. Software development in startup companies: the greenfield startup model. IEEE Transactions on Software Engineering, 42(6):585-604, 2015. 